\title{
Convexification Procedures and Decomposition Methods for Nonconvex Optimization Problems ${ }^{1}$
}

\author{
D. P. BERTSEKAS ${ }^{2}$ \\ Communicated by D. G. Luenberger
}

\begin{abstract}
In order for primal-dual methods to be applicable to a constrained minimization problem, it is necessary that restrictive convexity conditions are satisfied. In this paper, we consider a procedure by means of which a nonconvex problem is convexified and transformed into one which can be solved with the aid of primal-dual methods. Under this transformation, separability of the type necessary for application of decomposition algorithms is preserved. This feature extends the range of applicability of such algorithms to nonconvex problems. Relations with multiplier methods are explored with the aid of a local version of the notion of a conjugate convex function.
\end{abstract}

Key Words. Primal-dual methods, convexification procedures, decomposition methods, multiplier methods, local convex conjugate functions.

\section{Introduction}

Consider a constrained minimization problem of the form

$$
\begin{aligned}
& \text { minimize } f(x), \\
& \text { subject to } h(x)=0,
\end{aligned}
$$

where $f: R^{n} \rightarrow R, h: R^{n} \rightarrow R^{m}$ are given functions. Among several possible methods, one may attempt a numerical solution of this problem by means of a primal-dual method (see, e.g., Ref. 1, Chapter 13). In such methods, one forms the Lagrangian function $L: R^{n} \times R^{m} \rightarrow R$ defined by

$$
L(x, \lambda)=f(x)+\lambda^{\prime} h(x) .
$$

\footnotetext{
${ }^{1}$ This work was carried out at the Coordinated Science Laboratory, University of Illinois, Urbana, Illinois, and was supported by the National Science Foundation under Grant ENG $74-19332$.

${ }^{2}$ Associate Professor, Department of Electrical Engineering and Computer Science, Massachusetts Institute of Technology, Cambridge, Massachusetts.
} 
Subsequently, a dual functional $q$ is defined [perhaps, locally in a neighborhood of a Lagrange multiplier $\lambda^{*}$ of problem (1)] by means of

$$
q(\lambda)=\min _{x} L(x, \lambda),
$$

where the minimization is perhaps understood to be local in a neighborhood of a local minimum $x^{*}$ of problem (1). Then, one may employ a steepest ascent iferation of the form

$$
\lambda_{k+1}=\lambda_{k}+\alpha_{k} \nabla q\left(\lambda_{k}\right),
$$

where $\alpha_{k}>0$ is a scalar stepsize, or a Newton iteration of the form

$$
\lambda_{k+1}=\lambda_{k}-\left[\nabla^{2} q\left(\lambda_{k}\right)\right]^{-1} \nabla q\left(\lambda_{k}\right)
$$

in order to maximize the dual functional $q$. The gradient $\nabla q\left(\lambda_{k}\right)$ and Hessian $\nabla^{2} q\left(\lambda_{k}\right)$ under suitable assumptions exist and may be obtained via minimization of $L\left(\cdot, \lambda_{k}\right)$ (see Ref. 1). Related methods may be constructed for problems of the form

$$
\begin{array}{ll}
\text { minimize } & f(x), \\
\text { subject to } & x \in X, g_{j}(x) \leq 0, j=1, \ldots, r,
\end{array}
$$

where $g_{i}: R^{n} \rightarrow R$ and $X$ is a subset of $R^{n}$ (see, e.g. Refs. 2, 3, 4).

Methods of the type described above are particularly useful in separable problems having, for example, the form

$$
\begin{array}{ll}
\text { minimize } & \sum_{i=1}^{n} f_{i}\left(\xi_{i}\right), \\
\text { subject to } & \sum_{i=1}^{n} h_{i}\left(\xi_{i}\right)=0,
\end{array}
$$

where

$$
x=\left(\xi_{1}, \ldots, \xi_{n}\right)^{\prime} .
$$

In such problems, the minimization of the Lagrangian is decomposed into $n$ one-dimensional minimizations

$$
\min _{x} L\left(x, \lambda_{k}\right)=\sum_{i=1}^{n}\left\{\min _{\xi_{i}} f_{i}\left[\xi_{i}+\lambda_{k}^{\prime} h_{i}\left(\xi_{i}\right)\right]\right\}
$$

with considerable simplification resulting. This decomposition approach has been pioneered by Everett (Ref. 5) and has found considerable application in the solution of large-scale problems with separable structure.

One of the major drawbacks of primal-dual methods, which limits considerably their range of applicability, lies in the fact that the problem 
must have a convex structure (at least locally-near a solution), for otherwise it is either impossible to define a dual functional by means of (2) or else the maximal value of the dual functional is not equal to the optimal value of the original problem (i.e., a duality gap is present). Thus, in local versions of the theory for problem (1) (see Ref. 1), one needs the assumption that a local minimum-Lagrange multiplier pair $\left(x^{*}, \lambda^{*}\right)$ satisfies the second-order sufficiency conditions as well as the local convexity assumption

$$
z^{\prime} \nabla^{2} L\left(x^{*}, \lambda^{*}\right) z>0, \quad \forall z \neq 0
$$

In global versions of the theory for problem (5), it is necessary to assume that $X$ is a convex set and $f$ and $g_{j}$ are convex functions over $X$, and to make additional assumptions which ensure that there is no duality gap and that the dual functional is sufficiently differentiable in order for gradient-type methods to be applicable.

Now, it is possible to convexify problem (1) by considering the equivalent problem

$$
\begin{array}{ll}
\text { minimize } & f(x)+(c / 2)|h(x)|^{2}, \\
\text { subject to } & h(x)=0
\end{array}
$$

where $c>0$ is a scalar penalty parameter and $|\cdot|$ denotes the usual Euclidean norm. It is well known that, for $c$ sufficiently large, problem (6) has a locally convex structure provided $\left(x^{*}, \lambda^{*}\right)$ satisfy the second-order sufficiency assumptions. This follows from a lemma due to Debreu (Ref. 6). The well-known methods of multipliers may be viewed as primal-dual iterations of the form (3) or (4) applied to problem (6). We refer to Refs. 7 and 8 for analysis related to this viewpoint. While the convexification procedure described above has led to very useful general-purpose algorithms, it has the drawback that it precludes the straightforward application of decomposition algorithms for solving the corresponding dual problem, since the penalty term $(c / 2)|h(x)|^{2}$ does not have a separable structure even if the constraint function $h$ is separable. We mention, however, that the convexification procedure described above has been utilized in a recent paper by Stephanopoulos and Westerberg (Ref. 9) to construct a primal-dual method for separable nonconvex problems. No specific convergence and rate of convergence results have been given for the algorithm proposed in Ref. 9.

The purpose of this paper is to consider and analyze a different convexification procedure than the one above, which has the advantage that it preserves separable structure whenever it is already present. A simple way 
to describe our procedure for the case of the problem

$$
\begin{array}{ll}
\text { minimize } & f(x), \\
\text { subject to } & h(x)=0, g(x) \leq 0,
\end{array}
$$

is as follows.

We consider the problem

$$
\begin{array}{ll}
\text { minimize } & f(x)+(1 / 2 c)|y-x|^{2}, \\
\text { subject to } & h(x)=0, g(x) \leq 0, x \in R^{n}, y \in R^{n},
\end{array}
$$

where $c>0$ is some fixed scalar and $y$ represents a vector of additional variables. Clearly, a vector $x^{*}$ is a local minimum of the original problem (7) iff $\left(x^{*}, x^{*}\right)$ is a local minimum for problem (8). Now we may write problem (8) as

$$
\begin{array}{ll}
\text { minimize } & \varphi_{c}(y), \\
\text { subject to } & y \in R^{n},
\end{array}
$$

where the function $\varphi_{c}$ is defined by

$$
\varphi_{c}(y)=\min _{\substack{h(x)=0 \\ g(x) \leq 0}}\left\{f(x)+(1 / 2 c)|y-x|^{2}\right\},
$$

and the minimization above is understood to be local in a neighborhood of a local minimum $x^{*}$ of problem (7). It is easy to show that the minimization problem in (9) has a locally convex structure for $c$ small enough provided suitable second-order sufficiency conditions are satisfied at $x^{*}$. Thus, problem (9) may be solved by primal-dual methods. Furthermore, if the original problem (7) has separable structure, the same is true for the problem (9). Now, the function $\varphi_{c}$ of $(9)$ has $x^{*}$ as a local minimum and may be minimized by means of a steepest descent method, such as

$$
y_{k+1}=y_{k}-\alpha_{k} \nabla \varphi_{c}\left(y_{k}\right),
$$

where $\alpha_{k}$ is a stepsize parameter, or by means of Newton's method

$$
y_{k+1}=y_{k}-\left[\nabla^{2} \varphi_{c}\left(y_{k}\right)\right]^{-1} \nabla \varphi_{c}\left(y_{k}\right)
$$

It turns out that, under second-order sufficiency assumptions for $x^{*}$, both $\nabla \varphi_{c}$ and $\nabla^{2} \varphi_{c}$ exist within a sphere centered at $x^{*}$ for every $c>0$. Furthermore, this sphere can be made arbitrarily large by taking $c$ sufficiently large. These facts will be shown in the next section. At the same time, we will obtain convergence and rate of convergence results for iterations such as $(10)$ and (11). 
It is important to note that our approach bears a close relation to the method of multipliers. This relation is direct and easy to obtain whenever the function $\varphi: R^{n} \rightarrow(-\infty,+\infty)$ defined by

$$
\varphi(x)= \begin{cases}f(x), & \text { if } h(x)=0, g(x) \leq 0, \\ +\infty, & \text { otherwise }\end{cases}
$$

is convex and lower semicontinuous. Under these circumstances, $\varphi$ may be viewed as the ordinary dual functional of the concave programming problem

$$
\begin{array}{ll}
\text { maximize } & -\varphi^{*}(u), \\
\text { subject to } & -u=0,
\end{array}
$$

where $\varphi^{*}$ is the conjugate convex function of $\varphi$ given by

$$
\varphi^{*}(u)=\sup \left\{x^{\prime} u-\varphi(x)\right\} .
$$

By applying Fenchel's duality theorem and using (9), (12), it is easy to show that

$$
\begin{aligned}
\varphi_{c}(y) & =\inf _{x}\left\{\varphi(x)+(1 / 2 c)|y-x|^{2}\right\}=\sup _{u}\left\{-\varphi^{*}(u)+y^{\prime} u-(c / 2)|u|^{2}\right\} \\
& =-\inf _{u}\left\{\varphi^{*}(u)-y^{\prime} u+(c / 2)|u|^{2}\right\} .
\end{aligned}
$$

Thus $\varphi_{c}$ is the penalized dual functional which is optimized in the quadratic method of multipliers (see Refs. 10, 11) applied to problem (12). Furthermore, iterations (10) and (11) are first-order and second-order multiplier iterations (see Refs. 7, 8, 12) for solving problem (12), or equivalently for minimizing $\varphi_{c}$. The special case of iteration (10), where $\alpha=c$, is the original method of multipliers proposed by Hestenes and Powell. It has been analyzed exhaustively for the convex case by Rockafellar and Kort and Bertsekas (see Refs. 10,11,13), and more recently by Rockafellar (Refs. 14, 15 ) as a special case of the proximal point algorithm. The more general case, where the stepsize $\alpha_{k}$ is any scalar in the interval $[\delta, 2 c-\delta]$, where $\delta>0$ is some arbitrarily small scalar, has been analyzed by the author in Ref. 12 .

In the nonconvex case considered in this paper, the results available for multiplier methods cannot be directly invoked, since we do not have a conjugate convex function $\varphi^{*}$ and problem $(12)$ to work with. We bypass this difficulty by introducing in the Appendix the notion of a local conjugate convex function. Using this notion, we construct a problem similar to (12). Our algorithms are then shown to be multiplier methods for this problem, and their convergence properties follow from known results for these methods. 
The analysis throughout the paper is conducted in $n$-dimensional Euclidean space, denoted by $R^{n}$, and equipped with the usual norm, denoted by $|\cdot|$, i.e.,

$$
|x|=\left(\sum_{i=1}^{n} x_{i}^{2}\right)^{1 / 2} \quad \text { for } x=\left(x_{1}, \ldots, x_{n}\right)^{\prime} \in R^{n} .
$$

In our notation, all vectors will be considered as column vectors. A prime denotes transposition. For $\epsilon>0$ and $x \in R^{n}$, we denote by $S(x ; \epsilon)$ the open space centered at $x$ with radius $\epsilon$. For any function $h: R^{n} \rightarrow R$, we denote by $\nabla h$ and $\nabla^{2} h$ the gradient and Hessian matrix of $h$. For $h: R^{n} \rightarrow R^{m}, h=$ $\left(h_{1}, \ldots, h_{m}\right)^{\prime}$, we denote by $\nabla h$ the $n \times m$ matrix having as columns the gradients $\nabla h_{1}, \ldots, \nabla h_{m}$. For any $x \in R^{n}$, the notation $x \geq 0$ or $x \leq 0$ means that all coordinates of $x$ are nonnegative or nonpositive, respectively. We refer to $(x, \lambda, \mu)$ as a local minimum-Lagrange multiplier pair of a problem of the form (7) if $x$ is a local minimum and, together with $\lambda, \mu$, satisfies the first-order Kuhn-Tucker conditions for optimality (see Assumption 2.2 in the next section).

\section{Convergence Analysis}

Let $x^{*}$ be a local minimum of the problem

$$
\begin{array}{ll}
\text { minimize } & f(x), \\
\text { subject to } & h(x)=0, g(x) \leq 0,
\end{array}
$$

where $f: R^{n} \rightarrow R, \quad h: R^{n} \rightarrow R^{m}, \quad h=\left(h_{1}, \ldots, h_{m}\right)^{\prime}, \quad g: R^{n} \rightarrow R^{r}, \quad g=$ $\left(g_{1}, \ldots, g_{r}\right)^{\prime}$. Throughout the paper, we employ the following second-order sufficiency assumptions.

Assumption 2.1. The functions $f, h, g$ are twice continuously differentiable within an open sphere centered at $x^{*}$.

Assumption 2.2. The gradients $\nabla h_{i}\left(x^{*}\right), i=1, \ldots, m, \nabla g_{j}\left(x^{*}\right), j \in$ $A\left(x^{*}\right)$, where

$$
A\left(x^{*}\right)=\left\{j \mid g_{i}\left(x^{*}\right)=0, j=1, \ldots, r\right\}
$$

are linearly independent, and hence there exist unique Lagrange multiplier vectors

$$
\lambda^{*}=\left(\lambda_{1}^{*}, \ldots, \lambda_{m}^{*}\right)^{\prime} \in R^{m}, \quad \mu^{*}=\left(\mu_{1}^{*}, \ldots, \mu_{r}^{*}\right)^{\prime} \in R^{r},
$$


such that the following Kuhn-Tucker conditions hold:

$$
\begin{gathered}
\nabla f\left(x^{*}\right)+\nabla h\left(x^{*}\right) \lambda^{*}+\nabla g\left(x^{*}\right) \mu^{*}=0 \\
\mu^{*} \geq 0, \quad \mu_{j}^{*} g_{j}\left(x^{*}\right)=0, \quad j=1, \ldots, r .
\end{gathered}
$$

Assumption 2.3. Strict complementarity holds, i.e.,

$$
\mu_{i}^{*}>0 \quad \text { iff } \quad g_{j}\left(x^{*}\right)=0, \quad j=1, \ldots, r \text {. }
$$

Assumption 2.4. There holds

$$
z^{\prime}\left[\nabla^{2} f\left(x^{*}\right)+\sum_{i=1}^{m} \lambda_{i}^{*} \nabla^{2} h_{i}\left(x^{*}\right)+\sum_{j=1}^{r} \mu_{j}^{*} \nabla^{2} g_{j}\left(x^{*}\right)\right] z>0
$$

for all $z \in R^{n}$ such that $z \neq 0, \nabla h_{i}\left(x^{*}\right)^{\prime} z=0, i=1, \ldots, m, \nabla g_{j}\left(x^{*}\right)^{\prime} z=0$, $j \in A\left(x^{*}\right)$.

For any fixed $y \in R^{n}$ and $c>0$, consider the problem

$$
\begin{array}{ll}
\text { minimize } & f(x)+(1 / 2 c)|y-x|^{2}, \\
\text { subject to } & h(x)=0, g(x) \leq 0
\end{array}
$$

The necessary conditions for optimality for this problem are

$$
\begin{gathered}
\nabla f(x)+(1 / c)(x-y)+\nabla h(x) \lambda+\nabla g(x) \mu=0, \\
h(x)=0, \quad g(x) \leq 0, \quad \mu \geqslant 0, \quad \mu_{j} g_{j}(x)=0, \quad j=1, \ldots, r
\end{gathered}
$$

and may be viewed as a set of relations in $x, \lambda, \mu$ continuously parametrized by $y$. For $y=x^{*}$, we have the solution

$$
x=x^{*}, \quad \lambda=\lambda^{*}, \quad \mu=\mu^{*} .
$$

Consider the Jacobian matrix

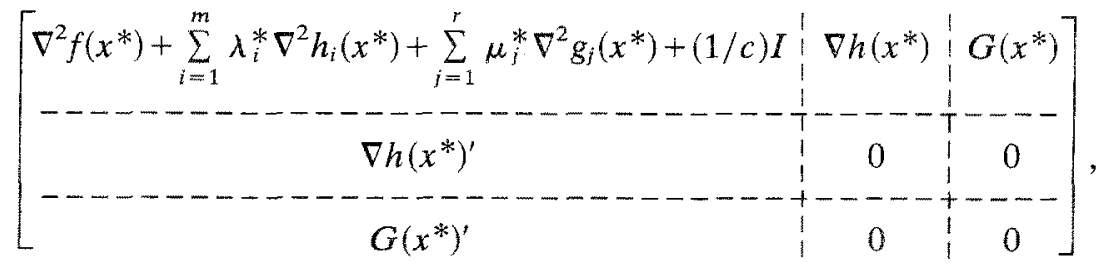

where $G\left(x^{*}\right)$ is the matrix having as columns $\nabla g_{j}\left(x^{*}\right), j \in A\left(x^{*}\right)$, and $I$ is the $n \times n$ identity matrix. It is easy to show that Assumptions 2.1-2.4 imply that this matrix is invertible for each $c>0$. It follows from the implicit function theorem that the system $(15),(16)$ has a unique solution $x(y, c), \lambda(y, c)$, 
$\mu(y, c)$, such that

$$
\begin{aligned}
& g_{j}[x(y, c)]=0, \quad \forall j \in A\left(x^{*}\right), \quad g_{j}[x(y, c)]<0, \quad \forall j \notin A\left(x^{*}\right), \\
& \mu_{j}(y, c)>0, \quad \forall j \in A\left(x^{*}\right), \quad \mu_{j}(y, c)=0, \quad \forall j \notin A\left(x^{*}\right),
\end{aligned}
$$

for each fixed $c>0$ and each $y$ in a sufficiently small neighborhood of $x^{*}$. This neighborhood depends on $c$. Furthermore, for $y$ sufficiently close to $x^{*}$, it can be seen that the vectors $x(y, c), \lambda(y, c), \mu(y, c)$ satisfy second-order sufficiency conditions for optimality for problem (14), and hence $x(y, c)$ is a strict local minimum for problem (14). Thus, we may define for $y \in S\left(x^{*} ; \epsilon\right)$, where $\epsilon>0$ is some scalar, the function

$$
\begin{aligned}
\varphi_{c}(y) & =f[x(y, c)]+(1 / 2 c)|y-x(y, c)|^{2} \\
& =\min _{\substack{h(x)=0 \\
g(x) \leq 0}}\left\{f(x)+(1 / 2 c)|y-x|^{2}\right\}
\end{aligned}
$$

where the minimization is local in the sense described above. The vector $x^{*}$ is clearly a local minimum of $\varphi_{c}$. Furthermore, we will show in Propositions 2.1 and 2.2 which follow that $\varphi_{c}$ is twice continuously differentiable in $S\left(x^{*} ; \epsilon\right)$ and that $\nabla \varphi_{c}, \nabla^{2} \varphi_{c}$ can be expressed in closed form in terms of $x(y, c), y, c$. Thus, one may employ the steepest descent iteration

$$
y_{k+1}=y_{k}-\alpha_{k} \nabla \varphi_{c}\left(y_{k}\right),
$$

where $\alpha_{k}>0$ is a scalar stepsize parameter, or Newton's iteration

$$
y_{k+1}=y_{k}-\left[\nabla^{2} \varphi_{c}\left(y_{k}\right)\right]^{-1} \nabla \varphi_{c}\left(y_{k}\right)
$$

for minimizing $\varphi_{c}$ locally within a neighborhood of the local minimum $y=x^{*}$.

It is possible to show that iterations (18) and (19) yield in the limit the vector $x^{*}$ provided the starting point $y_{0}$ is sufficiently close to $x^{*}$ and (in the case of steepest descent) the stepsize $\alpha_{k}$ is sufficiently small. These properties follow from well-known facts on gradient-type algorithms for unconstrained minimization and provide justification for employment of iterations (18) and (19). However, we can obtain considerably stronger algorithms and results by broadening our framework to include the possibility of changing the parameter $c$ from one iteration to the next and by allowing starting points $y_{0}$ which are arbitrarily far from $x^{*}$. The main results are described in the following two propositions, the proofs of which will be given in the next section. In each proposition, the function $\varphi_{c}$ of $(17)$ is redefined appropriately. Since we essentially deal with the same function in each proposition, we shall use a common notation for $\varphi_{c}$ and $x(y, c)$. Hopefully, this will not create any confusion to the reader. 
Proposition 2.1. Let $\gamma_{1}, \gamma_{2}$ be any two scalars with $0<\gamma_{1} \leq \gamma_{2}$. Then, there exists an $\epsilon>0$ (depending on $\gamma_{1}, \gamma_{2}$ ) such that, for all $c$ and $y$ with

$$
\gamma_{1} \leq c \leq \gamma_{2}, \quad\left|y-x^{*}\right|<\epsilon,
$$

problem (14) has a unique local minimum-Lagrange multiplier pair, denoted by $[x(y, c), \lambda(y, c), \mu(y, c)]$, within some open sphere centered at $\left(x^{*}, \lambda^{*}, \mu^{*}\right)$. The active constraints at $x(y, c)$ are the same as those at $x^{*}$, i.e., $g_{j}[x(y, c)]=0$, iff $j \in A\left(x^{*}\right)$. Furthermore, $\epsilon$ is such that the following hold true:

(i) The function $\varphi_{c}: S\left(x^{*} ; \epsilon\right) \rightarrow R$ defined for each $c \in\left[\gamma_{1}, \gamma_{2}\right]$ by

$$
\varphi_{c}(y)=f[x(y, c)]+(1 / 2 c)|y-x(y, c)|^{2}
$$

is twice continuously differentiable in $S\left(x^{*} ; \epsilon\right)$ and has $x^{*}$ as its unique minimum. The gradient of $\varphi_{c}$ is given by

$$
\nabla \varphi_{c}(y)=(1 / c)[y-x(y, c)] .
$$

The Hessian of $\varphi_{c}$ is the positive-definite matrix given by

$$
\nabla^{2} \varphi_{c}(y)=[c I+P(y, c)]^{-1}
$$

where $I$ is the $n \times n$ identity and $P(y, c)$ is the positive-semidefinite matrix given by

$$
P(y, c)=N^{-1}-N^{-1} F\left(F^{\prime} N^{-1} F\right)^{-1} F^{\prime} N^{-1} .
$$

The matrix $F$ above is the matrix having as columns the vectors $\nabla h_{i}[x(y, c)]$, $i=1, \ldots, m, \nabla g_{j}[x(y, c)], j \in A\left(x^{*}\right)$. The matrix $N$ equals

$$
L(y, c)=\nabla^{2} f[x(y, c)]+\sum_{i=1}^{m} \lambda_{i}(y, c) \nabla^{2} h_{i}[x(y, c)]+\sum_{j=1}^{r} \mu_{j}(y, c) \nabla^{2} g_{i}[x(y, c)]
$$

whenever $L(y, c)$ is invertible. Otherwise, $N$ equals any invertible matrix $\tilde{L}$ such that

$$
L(y, c) z=\tilde{L} z
$$

for all $z$ such that

$$
\nabla h_{i}[x(y, z)]^{\prime} z=0, \quad i=1, \ldots, m, \quad \nabla g_{j}[x(y, c)]^{\prime} z=0, \quad j \in A\left(x^{*}\right) .
$$

Note that, as shown in the proof, the matrix $P(y, c)$ is uniqely defined in this way.

(ii) Let $\left\{c_{k}\right\}$ be a sequence satisfying $\gamma_{1} \leq c_{k} \leq \gamma_{2}$ for all $k$, and let $\delta$ be any scalar such that $0<\delta \leq 1$. Consider the steepest descent iteration

$$
y_{k+1}=y_{k}-\alpha_{k} \nabla \varphi_{c_{k}}\left(y_{k}\right),
$$


where $\alpha_{k}$ satisfies for all $k$

$$
\delta c_{k} \leq \alpha_{k} \leq(2-\delta) c_{k} .
$$

Then, for every starting point $y_{0} \in S\left(x^{*} ; \epsilon\right)$, the sequence $\left\{y_{k}\right\}$ generated by (25) satisfies for all $k$

$$
\left|y_{k+1}-x^{*}\right| \leq r_{k}\left|y_{k}-x^{*}\right|
$$

where

$$
r_{k}=\sup _{\substack{y \in S\left(x^{*}, \epsilon\right) \\ i=1, \ldots, n}}\left|1-\alpha_{k} /\left[c_{k}+e_{i}\left(y, c_{k}\right)\right]\right|<1,
$$

and $e_{i}\left(y, c_{k}\right)$ denotes the ith eigenvalue of $P\left(y, c_{k}\right)$. Furthermore, $\left\{y_{k}\right\}$ converges to $x^{*}$.

(iii) Let $\left\{c_{k}\right\}$ be any sequence satisfying $\gamma_{1} \leq c_{k} \leq \gamma_{2}$ for all $k$, and consider the Newton iteration

$$
y_{k+1}=y_{k}-\left[\nabla^{2} \varphi_{c_{k}}\left(y_{k}\right)\right]^{-1} \nabla \varphi_{c_{k}}\left(y_{k}\right) .
$$

Then, there exists a scalar $\bar{\epsilon}$, with $0<\bar{\epsilon} \leq \epsilon$, such that, for every $y_{0} \in$ $S\left(x^{*} ; \bar{\epsilon}\right)$, the sequence $\left\{y_{k}\right\}$ generated by $(28)$ remains in $S\left(x^{*} ; \bar{\epsilon}\right)$ and converges to $x^{*}$. If, in addition, $\nabla^{2} f, \nabla^{2} h_{i}, i=1, \ldots, m, \nabla^{2} g_{j}, j \in A\left(x^{*}\right)$, are Lipschitz continuous in $S\left(x^{*} ; \bar{\epsilon}\right)$, then there exists a $B>0$ such that for all $k$

$$
\left|y_{k+1}-x^{*}\right| \leq B\left|y_{k}-x^{*}\right|^{2} .
$$

Proposition 2.2. Let $Y$ be any open sphere centered at $x^{*}$, and assume that $\nabla^{2} f, \nabla^{2} h_{i}, i=1, \ldots, m, \nabla^{2} g_{j}, j \in A\left(x^{*}\right)$, are Lipschitz continuous within a neighborhood of $x^{*}$. Then, there exists a scalar $\gamma \geq 0$ (depending on $Y$ ) such that, for all $c>\gamma$ and $y \in Y$, problem (14) has a unique local minimum-Lagrange multiplier pair, denoted by $[x(y, c), \lambda(y, c), \mu(y, c)]$, within some open sphere centered at $\left(x^{*}, \lambda^{*}, \mu^{*}\right)$. The active constraints at $x(y, c)$ are the same as those at $x^{*}$, i.e.,

$$
g_{j}[x(y, c)]=0
$$

iff $j \in A\left(x^{*}\right)$. Furthermore, $\gamma$ is such that the following hold true:

(i) The function $\varphi_{c}: Y \rightarrow R$ defined for each $c>\gamma$ by

$$
\varphi_{c}(y)=f[x(y, c)]+(1 / 2 c)|y-x(y, c)|^{2}
$$

is twice continuously differentiable in $Y$ and has $x^{*}$ as its unique minimum. 
The gradient and Hessian matrix of $\varphi_{c}$ are given by (21)-(23).

(ii) There exist scalars $M_{1}>0, M_{2}>0$, such that, for all $c>\gamma, y \in Y$, $\alpha>0$, there holds

$$
\begin{gathered}
\left|x(y, c)-x^{*}\right| \leq(1 / c) M_{1}\left|y-x^{*}\right|, \\
\left|y-\alpha \nabla \varphi_{c}(y)-x^{*}\right| \leq(1 / c)\left(|c-\alpha|+(\alpha / c) M_{1}\right)\left|y-x^{*}\right|, \\
\left|y-\left[\nabla^{2} \varphi_{c}(y)\right]^{-1} \nabla \varphi_{c}(y)-x^{*}\right| \leq\left(1 / c^{2}\right) M_{2}\left|y-x^{*}\right|^{2} .
\end{gathered}
$$

Propositions 2.1 and 2.2 provide related but different sets of results. In Proposition 2.1, essentially no restriction is made on the choice of the parameter $c$, since $\gamma_{1}$ and $\gamma_{2}$ are arbitrary positive scalars. However, the construction of $\varphi_{c}$ and the algorithmic results are local in nature, since $y$ is restricted within the sphere $S\left(x^{*} ; \epsilon\right)$ or $S\left(x^{*} ; \bar{\epsilon}\right)$, the radius of which depends on the choice of $c$. In Proposition 2.2, the situation is reversed. The domain of definition of $\varphi_{c}$ is the set $Y$ which can be taken arbitrarily large. However, both the construction of $\varphi_{c}$ and the algorithmic estimates (29)(31) are valid only for $c$ greater than the threshold value $\gamma$ which depends on the set $Y$.

Proposition 2.1 yields local convergence and rate of convergence results for the steepest descent and Newton iterations [parts (ii) and (iii)]. These results are valid for any sequence $\left\{c_{k}\right\}$ bounded above and bounded away from zero. In particular, $\left\{c_{k}\right\}$ can be taken so that problem (14) has a locally convex structure for every $k$. On the other hand, the iterations need not converge to $x^{*}$ if a starting point $y_{0}$ sufficiently close to $x^{*}$ is not available.

Part (ii) of Proposition 2.2 yields global convergence and rate of convergence results for both steepest descent and Newton iterations. Thus, the iteration

$$
y_{k+1}=x\left(y_{k}, c_{k}\right)
$$

converges to $x^{*}$ for an arbitrary starting point $y_{0} \in Y$, provided $c_{k} \geq \bar{\gamma}$ for all $k$, where $\bar{\gamma}$ is some scalar with

$$
\bar{\gamma}>\max \left\{M_{1}, \gamma\right\} \text {. }
$$

This follows immediately from (30). Furthermore, the rate of convergence is superlinear if $c_{k} \rightarrow \infty$. Notice that iteration (32) is the same as the steepest descent iteration (24) with $\alpha_{k}=c_{k}$ [compare (21), (25), and (32)]. From estimate (31), we obtain a similar global convergence result for the Newton iteration. The convergence rate is at least superlinear with order 2. Concerning the general steepest descent iteration

$$
y_{k+1}=y_{k}-\alpha_{k} \nabla \varphi_{c_{k}}\left(y_{k}\right),
$$


the reader may verify by using (30) that it is convergent from an arbitrary starting point $y_{0} \in Y$ provided we have, for all $k$,

$$
\delta c_{k} \leq \alpha_{k} \leq(2-\delta) c_{k}
$$

for some $\delta \in(0,1]$ and $c_{k} \geq \bar{\gamma}$, where

$$
\bar{\gamma}>\max \left\{(2-\delta) M_{1} / \delta, \gamma\right\} \text {. }
$$

Thus, the region of convergence for both steepest descent and Newton's method can be arbitrarily enlarged at the cost of having to take $c$ sufficiently large. This cost may be significant indeed, since, in order to convexify problem (14), small values of $c$ may be needed.

The conclusions from our results may be summarized by saying loosely that, if the amount of convexification induced is increased (i.e., $\mathrm{c}$ is reduced), the region of convergence of iterations (25) and (28) becomes smaller. The estimates (26), (27), (29)-(31) show also that the rate of convergence deteriorates as $\mathrm{c}$ is decreased. On the other hand, one can see that the conditioning of problem (14) is improved for small values of $c$. Thus, problem (14) becomes easier to solve algorithmically as $\mathrm{c}$ is decreased. The convergence behavior described above is reminiscent of the method of multipliers and has been verified in several computational examples, some of which will be presented in Section 4 .

\section{Proofs of the Propositions}

In order to simplify the presentation of the proofs, we restrict ourselves to the case where there are no inequality constraints and the problem is of the form

$$
\begin{array}{ll}
\text { minimize } & f(x), \\
\text { subject to } & h(x)=0 .
\end{array}
$$

The modifications required to prove the results for the general case are simple and are left to the reader.

We first note that, by Assumptions 2.1-2.4 and Definition 5.1 (see the Appendix), $f$ is $h$-locally convex at $x^{*}$. Let $p$ be the $h$-local conjugate of $f$ at $\left(x^{*}, \lambda^{*}\right)$. Then, $p$ is defined in a sphere $S(0 ; \delta), \delta>0$, and has the properties specified in Proposition 5.1. We have from the definition of $p[\mathrm{see}(55)]$ that, for all $u \in S(0 ; \delta)$,

$$
\begin{aligned}
u-\nabla f[x(u)]-\nabla h[x(u)] \lambda(u)=0, \quad h[x(u)]=0, \\
z^{\prime}\left[\nabla^{2} f[x(u)]+\sum_{i=1}^{m} \lambda_{i}(u) \nabla^{2} h_{i}[x(u)]\right] z>0, \quad \forall z \neq 0, \quad \nabla h[x(u)] z^{\prime}=0,
\end{aligned}
$$


and $x(u), \lambda(u)$ are unique vectors satisfying the above relations within $S\left(x^{*} ; \sigma\right)$ and $S\left(\lambda^{*} ; \sigma\right)$, respectively, where $\sigma>0$ is some scalar. Furthermore,

$$
\begin{gathered}
x(0)=x^{*}, \quad \lambda(0)=\lambda^{*}, \\
p(u)=u^{\prime} x(u)-f[x(u)], \quad \forall u \in S(0 ; \delta), \\
\nabla p(u)=x(u), \quad \forall u \in S(0 ; \delta) .
\end{gathered}
$$

Consider the problem

$$
\begin{array}{ll}
\text { minimize } & p(u), \\
\text { subject to } & -u=0, \quad u \in S(0 ; \delta) .
\end{array}
$$

By (35) and (36), the optimal value of this problem is $-f\left(x^{*}\right)$; and by (35) and (37), the associated Lagrange multiplier is

$$
\nabla p(0)=x(0)=x^{*}
$$

We will show that the algorithms proposed are multiplier methods for solving problem (38), of the type considered in Refs. 7, 8, 16. The following lemma plays a key role in this respect.

Lemma 3.1. If the problem

$$
\begin{array}{ll}
\text { minimize } & p(u)-y^{\prime} u+(c / 2)|u|^{2}, \\
\text { subject to } & u \in S(0 ; \delta),
\end{array}
$$

has a unique minimizing point, denoted by $u(y, c)$, then the problem

$$
\begin{array}{ll}
\text { minimize } & f(x)+(1 / 2 c)|y-x|^{2}, \\
\text { subject to } & h(x)=0, \quad x \in S\left(x^{*} ; \sigma\right),
\end{array}
$$

has a unique minimizing point, denoted by $x(y, c)$, and we have

$$
\begin{gathered}
x(y, c)=y-c u(y, c), \\
p[u(y, c)]-y^{\prime} u(y, c)+(c / 2)|u(y, c)|^{2}=-f[x(y, c)]-(1 / 2 c)|y-x(y, c)|^{2}
\end{gathered}
$$

Proof. Using (34), define

Then,

$$
x(y, c)=x[u(y, c)], \quad \lambda(y, c)=\lambda[u(y, c)] .
$$

$$
x(y, c) \in S\left(x^{*} ; \sigma\right), \quad \lambda(y, c) \in S\left(\lambda^{*} ; \sigma\right) .
$$

We have

$$
\nabla p[u(y, c)]-y+c u(y, c)=0
$$


and, using (37) and (43), we obtain (41). A straightforward calculation using (36), (41), (43) also yields (42). From (34), (41), (43), we obtain

$$
\nabla f[x(y, c)]+(1 / c)[x(y, c)-y]+\nabla h[x(y, c)] \lambda(y, c)=0
$$

from which it follows that $x(y, c)$ is a minimizing point of problem (40). It is a unique minimizing point, in view of the uniqueness of $x(u)$ and $\lambda(u)$ within $S\left(x^{*} ; \sigma\right)$ and $S\left(\lambda^{*} ; \sigma\right)$ satisfying $(34-1)$.

The proofs of Propositions 2.1 and 2.2 follow by straightforward application of Lemma 3.1, Proposition 5.1, and known results on multiplier methods given in Ref. 7 (Propositions 1, 2, 5 and Corollary 2.1), Ref. 8 (Proposition 6), and Ref. 16 (Proposition 1). The details are left to the reader.

\section{Computational Aspects and Results}

We now consider a number of topics of computational nature related to our methods.

Stepsize Selection for Steepest Descent. As shown in Propositions 2.1 and 2.2 , the steepest descent iteration takes the form

$$
y_{k+1}=y_{k}-\left(\alpha_{k} / c_{k}\right)\left[y_{k}-x\left(y_{k}, c_{k}\right)\right]=\left[\left(c_{k}-\alpha_{k}\right) / c_{k}\right] y_{k}+\left(\alpha_{k} / c_{k}\right) x\left(y_{k}, c_{k}\right)
$$

The stepsize $\alpha_{k}$ can take values in $\left[\delta c_{k},(2-\delta) c_{k}\right]$, where $\delta \in(0,1]$ is an arbitrarily small scalar. In particular, when $\alpha_{k}=c_{k}$ and the iteration takes the form $y_{k+1}=x\left(y_{k}, c_{k}\right)$, convergence is assured as shown in Propositions 2.1 and 2.2. On the other hand, one would like to select the stepsize in a way that accelerates convergence of the method. Ideally, one would like to find the stepsize which minimizes $\varphi_{c_{k}}(y)$ along the direction of search. It is possible to show that the minimizing stepsize is always greater or equal to $c_{k}$. To see this, consider the first derivative of the scalar function $\Phi_{k}$ defined by

$$
\Phi_{k}(\alpha)=\varphi_{c_{k}}\left[y_{k}-\alpha \nabla \varphi_{c_{k}}\left(y_{k}\right)\right]
$$

at the point $c_{k}$. We have, from the chain rule,

$$
d \Phi_{k}\left(c_{k}\right) / d \alpha=-\nabla \varphi_{c_{k}}\left(y_{k}\right)^{\prime} \nabla \varphi_{c_{k}}\left[x\left(y_{k}, c_{k}\right)\right]
$$


By using Taylor's theorem and Eqs. (21), (22), we obtain

$$
\begin{aligned}
\nabla \varphi_{c_{k}}\left[x\left(y_{k}, c_{k}\right)\right]= & \nabla \varphi_{c_{k}}\left(y_{k}\right)+\int_{0}^{1} \nabla^{2} \varphi_{c_{k}}\left[y_{k}+t\left[x\left(y_{k}, c_{k}\right)-y_{k}\right]\right] d t\left[x\left(y_{k}, c_{k}\right)-y_{k}\right] \\
= & \left.\left\{I-c_{k} \int_{0}^{1} \nabla^{2} \varphi_{c_{k}}\left[y_{k}+t\left[x\left(y_{k}, c_{k}\right)-y_{k}\right]\right] d t\right\} \nabla \varphi_{c_{k}}{ }_{k}\right) \\
= & \left\{I-\int_{0}^{1}\left[I+\left(1 / c_{k}\right) P\left[y_{k}+t\left[x\left(y_{k}, c_{k}\right)-y_{k}\right], c_{k}\right]\right]^{-1} d t\right\} \\
& \times \nabla \varphi_{c_{k}}\left(y_{k}\right) .
\end{aligned}
$$

Since the matrix $P$ is positive semidefinite, it follows that the matrix within braces above is also positive semidefinite. Hence, from (44), (45), we obtain

$$
d \Phi_{k}\left(c_{k}\right) / d \alpha \leq 0 .
$$

Since $\Phi_{k}(\alpha)$ is also convex within the region of its definition and

$$
d \Phi_{k}(0) / d \alpha \leq 0,
$$

it follows that the stepsize $\alpha_{k}$ which minimizes $\Phi_{k}(\alpha)$ is always greater or equal to $c_{k}$. We note that a similar result has been proved for multiplier methods (see Ref. 7, Section 5). Since it is computationally inconvenient to perform a one-dimensional minimization of $\varphi_{c}(y)$ along the steepest descent direction, an alternative is to minimize $f$ over the set of points which correspond to stepsizes in the interval $\left[c_{k}, 2 c_{k}-\delta\right]$ and in addition are feasible. In other words, we propose to determine $\alpha_{k}$ from

$$
f\left[y_{k}-\alpha_{k} \nabla \varphi_{c_{k}}\left(y_{k}\right)\right]=\min _{\alpha \in M_{k}} f\left[y_{k}-\alpha \nabla \varphi_{c_{k}}\left(y_{k}\right)\right],
$$

where $M_{k}$ is the subset of the real line given by

$$
M_{k}=\left\{\alpha \mid \alpha \in\left[c_{k},(2-\delta) c_{k}\right], h\left[y_{k}-\alpha \nabla \varphi_{c_{k}}\left(y_{k}\right)\right]=0, g\left[y_{k}-\alpha \nabla \varphi_{c_{k}}\left(y_{k}\right)\right] \leq 0\right\} .
$$

Notice that $M_{k}$ always contains $c_{k}$, since we have

$$
y_{k}-c_{k} \nabla \varphi_{c_{k}}\left(y_{k}, c_{k}\right)=x\left(y_{k}, c_{k}\right)
$$

and

$$
h\left[x\left(y_{k}, c_{k}\right)\right]=0, \quad g\left[x\left(y_{k}, c_{k}\right)\right] \leq 0 .
$$

The minimization in (46) is simplified greatly when the feasible set $\{x \mid h(x)=0, g(x) \leq 0\}$ is convex, in which case the set $M_{k}$ of (47) is a closed interval. It is also possible to perform the minimization in (46) approximately by means, for example, of a few iterations of a quadratic or cubic 
interpolation procedure. Similar procedures for choosing the stepsize have been proposed for the method of multipliers by the author in Refs. 7, 12, and encouraging computational results have been given. We provide below some examples which demonstrate the convergence properties of the steepest descent method and show that choosing the stepsize via (46), (47) can result in significant computational savings.

Example 4.1. Consider the problem

$$
\begin{array}{ll}
\text { minimize } & -\xi_{1} \xi_{2}, \\
\text { subject to } & \xi_{1}+4 \xi_{2}-1=0,
\end{array}
$$

with optimal solution

$$
\xi_{1}^{*}=0.5, \quad \xi_{2}^{*}=0.125 .
$$

The optimal solution of the problem

$$
\begin{array}{ll}
\text { minimize } & -\xi_{1} \xi_{2}+(1 / 2 c)\left\{\left|y_{1}-\xi_{1}\right|^{2}+\left|y_{2}-\xi_{2}\right|^{2}\right\}, \\
\text { subject to } & \xi_{1}+4 \xi_{2}-1=0,
\end{array}
$$

is given by

$$
\begin{aligned}
& \xi_{1}(y, c)=\left(4 c+1+16 y_{1}-4 y_{2}\right) /(8 c+17), \\
& \xi_{2}(y, c)=\left(c+4-4 y_{1}+y_{2}\right) /(8 c+17) .
\end{aligned}
$$

We provide in Table 1 the results of the steepest descent iteration

$$
y_{1}^{k+1}=\xi_{1}\left(y^{k}, c_{k}\right), \quad y_{2}^{k+1}=\xi_{2}\left(y^{k}, c_{k}\right),
$$

corresponding to the stepwise $\alpha_{k}=c_{k}$ for the cases where $c_{k} \equiv 1$ and $c_{k} \equiv 10$.

In Table 2, we show the results of the steepest descent iteration, where the stepsize $\alpha_{k}$ was chosen via the minimization rule of (69), (70), where $\delta=10^{-8}$. These results are considerably more favorable than those of Table 1. We note however that, when the feasible set is a one-dimensional manifold, as it is in this problem, the minimization stepsize rule is much more effective than in problems where the feasible set is of dimension greater than one. Our next example is of this type.

Example 4.2. This problem was designed specifically to test the effectiveness of the minimization stepsize rule (46), (47) versus the stepsize $\alpha_{k}=c_{k}$. The problem is

$$
\operatorname{minimize} f(x)=(1 / 2) \sum_{i=1}^{n} i x_{i}^{2},
$$


Table 1. Steepest descent with stepsize $\alpha_{k}=c_{k}$.

\begin{tabular}{rcccc}
\hline & \multicolumn{3}{c}{$c_{k} \equiv 1$} & \multicolumn{2}{c}{$c_{k} \equiv 10$} \\
\cline { 2 - 5 }$k$ & $y_{1}^{k}$ & $y_{2}^{k}$ & $y_{1}^{k}$ & $y_{2}^{k}$ \\
\hline 0 & 0.00000 & 0.00000 & 0.00000 & 0.00000 \\
1 & 0.20000 & 0.20000 & 0.42268 & 0.14432 \\
2 & 0.29600 & 0.17600 & 0.48645 & 0.12838 \\
3 & 0.36128 & 0.15968 & 0.49762 & 0.12559 \\
4 & 0.40567 & 0.14858 & 0.49958 & 0.12510 \\
5 & 0.43585 & 0.14103 & 0.49992 & 0.12501 \\
6 & 0.45638 & 0.13590 & 0.49999 & 0.12500 \\
7 & 0.47034 & 0.13241 & & \\
8 & 0.47983 & 0.13004 & & \\
9 & 0.48628 & 0.12842 & & \\
10 & 0.49067 & 0.12733 & & \\
11 & 0.49366 & 0.12658 & & \\
\hline
\end{tabular}

subject to $x \in R^{n}$. This problem is unconstrained. Our results however apply, with trivial notational changes, to such a problem as well. By straightforward calculation, one obtains that the steepest descent iteration is given by

$$
y_{k+1}^{i}=\left\{\left[i\left(c_{k}-\alpha_{k}\right)+1\right] /\left(i c_{k}+1\right)\right\} y_{k}^{i}, \quad i=1, \ldots, n .
$$

The minimizing stepsize can be calculated to be

$$
\alpha_{k}=\left[\sum_{i=1}^{n}\left(i y_{k}^{i}\right)^{2}\right] / \sum_{i=1}^{n}\left(i y_{k}^{i}\right)^{2}\left[i /\left(i c_{k}+1\right)\right]
$$

For the values of $c_{k}$ and $y_{0}$ used, there holds $\alpha_{k} \in\left[c_{k}, 1.9 c_{k}\right]$. Tables 3 and 4 below show the sequences $\left\{f\left(y_{k}\right)\right\}$ generated for $c_{k} \equiv 1, c_{k} \equiv 10, n=3,10$,

Table 2. Steepest descent with minimizing stepsize [see (46), (47)].

\begin{tabular}{ccccc}
\hline & \multicolumn{3}{c}{$c_{k} \equiv 1$} & \multicolumn{2}{c}{$c_{k} \equiv 10$} \\
\cline { 2 - 5 }$k$ & $y_{1}^{k}$ & $y_{2}^{k}$ & $y_{1}^{k}$ & $y_{2}^{k}$ \\
\hline 0 & 0.00000 & 0.00000 & 0.00000 & 0.00000 \\
1 & 0.20000 & 0.20000 & 0.42268 & 0.14432 \\
2 & 0.39200 & 0.15200 & 0.50000 & 0.12500 \\
3 & 0.46112 & 0.13472 & & \\
4 & 0.48600 & 0.12850 & & \\
5 & 0.49496 & 0.12626 & & \\
6 & 0.49818 & 0.12545 & & \\
\hline
\end{tabular}




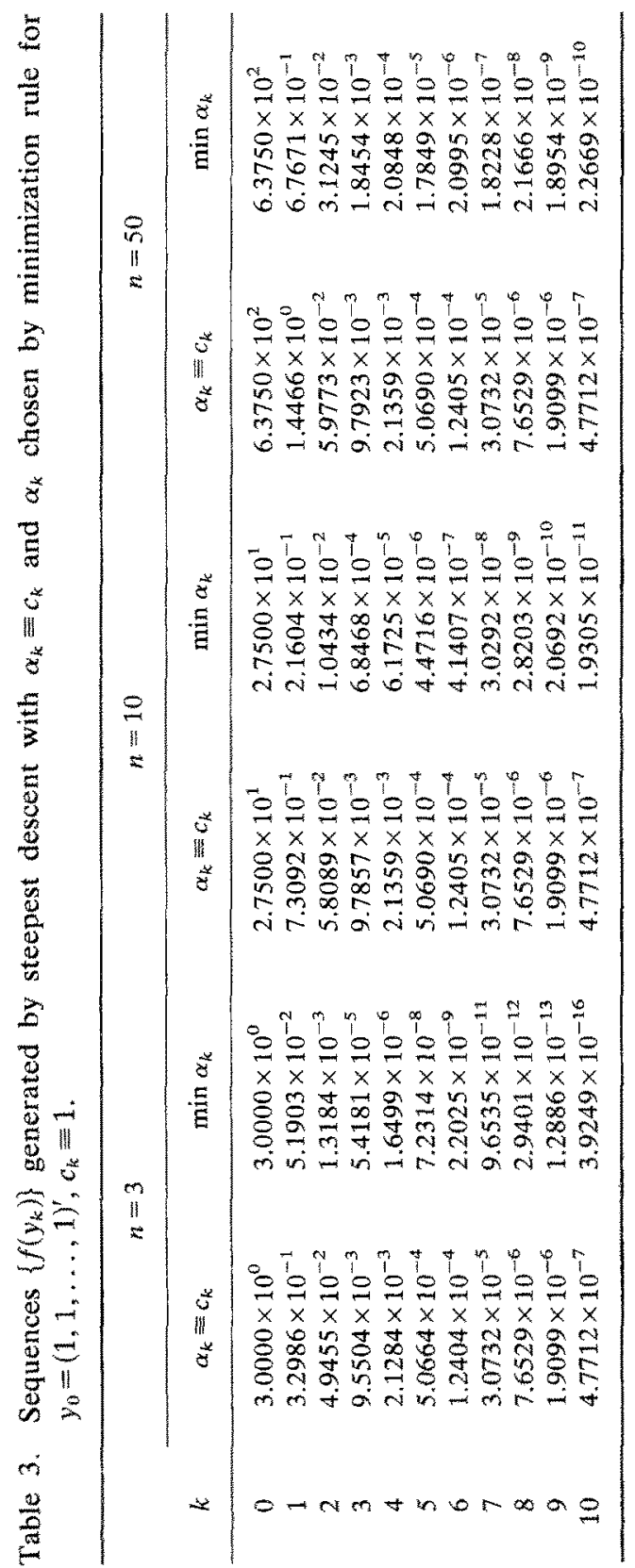




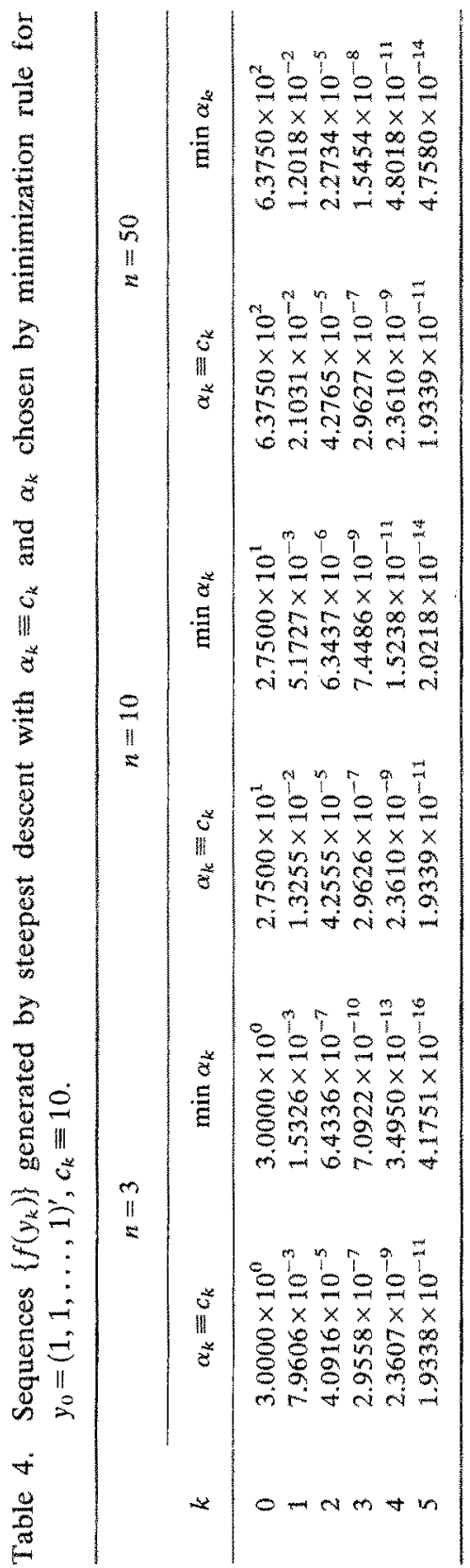


$50, \alpha_{k} \equiv c_{k}$, and $\alpha_{k}$ chosen by minimization $\left(\min \alpha_{k}\right)$. The initial vector $y_{0}$ was

$$
y_{0}=(1,1, \ldots, 1)^{\prime} .
$$

Very similar results were also obtained for

$$
y_{0}=(1,1 / 2, \ldots, 1 / n)^{\prime} \text {. }
$$

Newton Iteration for Separable Problems. Since a primary motivation of our algorithms has been the solution of large-scale separable problems, it is worthwhile to point out that the Newton iteration for such problems can often be carried out in a computationally efficient manner. Indeed, consider the separable problem

$$
\begin{array}{ll}
\text { minimize } & \sum_{i=1}^{n} f_{i}\left(x_{i}\right), \\
\text { subject to } & \sum_{i=1}^{n} h_{i}\left(x_{i}\right)=0,
\end{array}
$$

where

$$
x=\left(x_{1}, \ldots, x_{n}\right)^{\prime}
$$

and, for each $i, f_{i}: R \rightarrow R, h_{i}: R \rightarrow R^{m}$. The Newton iteration (28) takes the form

$$
y_{k+1}=y_{k}-\left[I+\left(1 / c_{k}\right) P\left(y_{k}, c_{k}\right)\right]\left[y_{k}-x\left(y_{k}, c_{k}\right)\right],
$$

where $P(y, c)$ is given by (23)-(25), i.e.,

$$
P(y, c)=N^{-1}-N^{-1} F\left(F^{\prime} N^{-1} F\right)^{-1} F^{\prime} N^{-1} .
$$

The matrix $N$ can be taken to be equal to $L(y, c)$, the Hessian of the Lagrangian function, whenever $L(y, c)$ is invertible. Let us assume that $i(y, c)$ is indeed invertible. Then, the computation in (49) is greatly facilitated, since $L(y, c)$ is diagonal, and hence $N^{-1}$ is immediately available. The main computational difficulty in (49) is to compute

$$
d_{k}=\left(F^{\prime} N^{-1} F\right)^{-1} F^{\prime} N^{-1}\left[y_{k}-x\left(y_{k}, c_{k}\right)\right] .
$$

This can be done by solving the system of $m$ linear equations

$$
\left(F^{\prime} N^{-1} F\right) d_{k}=F^{\prime} N^{-1}\left[y_{k}-x\left(y_{k}, c_{k}\right)\right] \text {. }
$$

The dimension of this system is $\mathrm{m}$ and is often small, even if the dimension $\mathrm{n}$ of the problem is very large. 
Frequently separable problems are of the form

$$
\begin{array}{ll}
\text { minimize } & \sum_{i=1}^{n} f\left(x_{i}\right), \\
\text { subject to } & \sum_{i=1}^{n} h_{i}\left(x_{i}\right)=0, \\
& \alpha_{i} \leq x_{i} \leq \beta_{i}, \quad i=1, \ldots, n .
\end{array}
$$

During the algorithm, we generate sequences $\left\{y_{k}\right\}$ and $\left\{x\left(y_{k}, c_{k}\right)\right\}$. According to Propositions 2.1 and 2.2 , the inequality constraints that are active at $x\left(y_{k}, c_{k}\right)$ are the same as those that are active at $x^{*}$. Let $A\left[x\left(y_{k}, c_{k}\right)\right]$ be the set of indices of coordinates of $x\left(y_{k}, c_{k}\right)$ which equal either the corresponding lower bound or the corresponding upper bound, i.e.,

$$
A\left[x\left(y_{k}, c_{k}\right)\right]=\left\{i \mid x_{i}\left(y_{k}, c_{k}\right)=\alpha_{i} \text { or } x_{i}\left(y_{k}, c_{k}\right)=\beta_{i}\right\} .
$$

Then, it is possible to prove that the rows and columns of $P\left(y_{k}, c_{k}\right)$ corresponding to indices in $A\left[x\left(y_{k}, c_{k}\right)\right]$ are zero. Thus, if indices are reordered so that, for some index $s$, we have

$$
A\left[x\left(y_{k}, c_{k}\right)\right]=\{i \mid s+1 \leq i \leq n\},
$$

then $P\left(y_{k}, c_{k}\right)$ has the form

$$
P\left(y_{k}, c_{k}\right)=\left[\begin{array}{c:c}
\tilde{N}^{-1}-\tilde{N}^{-1} \tilde{F}\left(\tilde{F}^{\prime} \tilde{N}^{-1} \tilde{F}\right)^{-1} \tilde{F}^{\prime} \tilde{N}^{-1} & 0 \\
\hdashline 0 & 0
\end{array}\right]
$$

where $\tilde{N}$ represents the Hessian of the Lagrangian with respect to the first $s$ coordinates of $x$ and $\tilde{F}$ is the $s \times n$ matrix with $(i, j)$ th element equal to

$$
\begin{gathered}
\partial h_{i}^{i}\left[x_{i}\left(y_{k}, c_{k}\right)\right] / \partial x_{i}, \quad i=1, \ldots, s, \quad j=1, \ldots, m, \\
\text { where } h_{i}=\left(h_{i}^{1}, \ldots, h_{i}^{m}\right)^{\prime} .
\end{gathered}
$$

In view of these facts, it can be seen that the Newton iteration (49) can be carried out quite easily and involves again the solution of a system of $m$ linear equations (rather than $n$ ), where $m$ is the number of equality constraints in problem (50). Similar simplifications occur when problem (50) involves in addition separable inequality constraints of the form

$$
\sum_{i=1}^{n} g_{i}\left(x_{i}\right) \leq 0
$$

In conclusion, if $L(y, c)$ is invertible, the Newton iteration for problem (49) or (50) requires the solution of a system of linear equations of dimension $\mathrm{m}$ (the number of equality constraints), rather than $\mathrm{n}$. 
Inexact Minimization. In our analysis and algorithms, we require that the exact value of the local minimum $x(y, c)$ of the problem

$$
\begin{array}{ll}
\text { minimize } & f(x)+(1 / 2 c)|y-x|^{2}, \\
\text { subject to } & h(x)=0, g(x) \leq 0,
\end{array}
$$

be available. In practice, the minimization above will be carried out only approximately. Not only will the algorithm become computationally implementable in this way, but, if experience with the method of multipliers can serve as a guide, one expects that inexact minimization should result in considerable computational savings. A possible scheme is to determine at the $k$ th iteration, in place of $x\left(y_{k}, c_{k}\right), \lambda\left(y_{k}, c_{k}\right), \mu\left(y_{k}, c_{k}\right)$, a vector $x_{k}$ satsifying, together with the Lagrange multiplier vectors $\lambda_{k}$ and $\mu_{k}$, the relations

$$
\begin{gathered}
\left|\nabla f\left(x_{k}\right)+\left(1 / c_{k}\right)\left(x_{k}-y_{k}\right)+\nabla h\left(x_{k}\right) \lambda_{k}+\nabla g\left(x_{k}\right) \mu_{k}\right| \leq\left(\gamma_{k} / c_{k}\right) \min \left\{\left|x_{k}-y_{k}\right|, 1\right\}, \\
\max _{\substack{i=1, \ldots, m \\
j=1, \ldots, r}}\left\{\left|h_{i}\left(x_{k}\right)\right|, g_{j}\left(x_{k}\right)\right\} \leq\left(\gamma_{k} / c_{k}\right) \min \left\{\left|x_{k}-y_{k}\right|, 1\right\}, \\
\max _{j=1, \ldots, r}\left|\mu_{k}^{j} g_{j}\left(x_{k}\right)\right| \leq\left(\gamma_{k} / c_{k}\right) \min \left\{\left|x_{k}-y_{k}\right|, 1\right\}
\end{gathered}
$$

where $\left\{\gamma_{k}\right\}$ is a nonincreasing nonnegative sequence converging to zero. In this way, the minimization is inexact, but becomes progressively more accurate. While we expect that algorithms employing a judicious scheme for inexact minimization should be computationally more efficient than algorithms with exact minimization, we have conducted no computational experiments to test this conjecture.

\section{Appendix: Local Conjugate Convex Functions}

Definition 5.1. Given a function $f: R^{n} \rightarrow R$ and a mapping $h: R^{n} \rightarrow$ $R^{m}, h=\left(h_{1}, \ldots, h_{m}\right)^{\prime}$, we say that $f$ is $h$-locally convex at a point $\bar{x} \in R^{n}$ if $h(\bar{x})=0, f$ and $h$ are twice continuously differentiable in a neighborhood of $\bar{x}$, the gradients $\nabla h_{1}(\bar{x}), \ldots, \nabla h_{m}(\bar{x})$ are linearly independent, and there exists a vector $\bar{\lambda}=\left(\bar{\lambda}_{1}, \ldots, \bar{\lambda}_{m}\right)^{\prime} \in R^{m}$ such that

$$
z^{\prime}\left[\nabla^{2} f(\bar{x})+\sum_{i=1}^{m} \bar{\lambda}_{i} \nabla^{2} h_{i}(\bar{x})\right]>0, \quad \forall z \neq 0, \quad \nabla h(\bar{x})^{\prime} z=0 .
$$

Let $f$ be $h$-locally convex at $\bar{x} \in R^{n}$, let $\bar{\lambda}$ satisfy (51), and consider the vector

$$
\bar{u}=\nabla f(\bar{x})+\nabla h(\bar{x}) \bar{\lambda} .
$$


Consider also, for fixed $u$, the problem

$$
\begin{array}{ll}
\text { maximize } & u^{\prime} x-f(x), \\
\text { subject to } & h(x)=0
\end{array}
$$

The standard second-order sufficiency conditions for this problem (see Ref. 1, p. 226) are

$$
\begin{gathered}
u-\nabla f(x)-\nabla h(x) \lambda=0, \quad h(x)=0, \\
z^{\prime}\left[\nabla^{2} f(x)+\sum_{i=1}^{m} \lambda_{i} \nabla^{2} h_{i}(x)\right] z>0, \quad \forall z \neq 0, \quad \nabla h(x)^{\prime} z=0 .
\end{gathered}
$$

The vectors $\bar{u}, \bar{x}, \bar{\lambda}$ satisfy conditions (54). From the implicit function theorem, it follows that there exist scalars $\delta>0$ and $\sigma>0$ such that, for every $u \in S(\bar{u} ; \delta)$, problem (53) has a unique local maximum within a sphere $S(\bar{x} ; \sigma)$, denoted by $x(u)$, and a unique associated Lagrange multiplier vector within $S(\bar{\lambda} ; \sigma)$, denoted by $\lambda(u)$, and satisfying, for all $u \in S(\bar{u} ; \delta)$,

$$
\begin{gathered}
u-\nabla f[x(u)]-\nabla h[x(u)] \lambda(u)=0, \quad h[x(u)]=0, \\
z^{\prime}\left[\nabla^{2} f[x(u)]+\sum_{i=1}^{m} \lambda_{i}(u) \nabla^{2} h_{i}[x(u)]\right] z>0, \quad \forall z \neq 0, \quad \nabla h[x(u)]^{t} z=0
\end{gathered}
$$

Furthermore, the vectors $\nabla h_{i}[x(u)], i=1, \ldots, m$, are linearly independent for all $u \in S(\bar{u} ; \delta)$. We define the h-local convex conjugate of $f$ at $(\bar{x}, \bar{\lambda})$ by

$$
p(u)=u^{\prime} x(u)-f[x(u)], \quad \forall u \in S(\tilde{u} ; \delta) .
$$

In order to gain better understanding of the nature of the local convex conjugate function, consider the set $S$ of all $u \in R^{n}$ around which a conjugate can be defined locally:

$$
\begin{aligned}
S= & \{u \mid u=\nabla f(x)+\nabla h(x) \lambda \text { for some }(x, \lambda) \text { with } h(x)=0, \\
& z^{\prime}\left[\nabla^{2} f(x)+\sum_{i=1}^{m} \lambda_{i} \nabla^{2} h_{i}(x)\right] z>0, \forall z \neq 0, \nabla h(x)^{\prime} z=0, \\
& \text { and } \left.\nabla h_{1}(x), \ldots, \nabla h_{m}(x) \text { linearly independent }\right\} .
\end{aligned}
$$

We first observe that $S$ is an open set. This can be seen from the fact that, if $\tilde{u} \in S$ and an $h$-local convex conjugate at $(\bar{x}, \bar{\lambda})$ is defined with $S(\tilde{u} ; \delta)$, then for every $u \in S(\bar{u} ; \delta)$ the vectors $x(u), \lambda(u)$ satisfy $(55)$, and hence $S(\vec{u} ; \delta) \subset S$. Now, to each vector $u \in S$ there may correspond more than one pair $(x, \lambda)$ satisfying $(55)$, and the value of the conjugate $p$ at $u$ will depend 
on the corresponding pair $(x, \lambda) .^{3}$ Thus, a perhaps more appropriate notation for $p$, and indeed an alternative (and equivalent) definition, would be to set

$$
p(u ; x, \lambda)=u^{\prime} x-f(x),
$$

for all $u \in S$, and $(x, \lambda)$ satisfying (54). This completely specifies the local conjugate for all points where it can be defined. In our definition (56), the dependence on $(x, \lambda)$ is suppressed, since $p$ is defined only locally within $S(\bar{u} ; \delta)$, rather than over the whole set $S$. In the case where to each $u \in S$ there corresponds a unique pair $[x(u), \lambda(u)]$ satisfying $(55)$, we have

$$
p(u)=u^{\prime} x(u)-f[x(u)], \quad \forall u \in S,
$$

and specification of $u$ determines $x(u)$, and hence also $p(u)$. We give two examples that may be helpful in clarifying this situation.

Example 5.1. For $n=2$, let

$$
f(x)=0, \quad h(x)=h\left(x_{1}, x_{2}\right)=(1 / 3) x_{1}^{3}-x_{2} .
$$

Let $\bar{x}=\left(\bar{x}_{1}, \bar{x}_{2}\right)^{\prime}$ be any vector satisfying

$$
(1 / 3) \bar{x}_{1}^{3}=\bar{x}_{2} \text {. }
$$

Then, for $z=\left(z_{1}, z_{2}\right)^{\prime},(51)$ is written as

$$
2 \bar{\lambda} \bar{x}_{1} z_{1}^{2}>0, \quad \forall z \neq 0, \quad \bar{x}_{1}^{2} z_{1}-z_{2}=0 .
$$

The above relation is satisfied for all $(\bar{x}, \bar{\lambda})$ with $\bar{\lambda} \bar{x}_{1}>0$. The $h$-local conjugate of $\mathrm{f}$ can be defined locally at each point $\bar{u}$ of the form

$$
\bar{u}=\left[\begin{array}{c}
\bar{\lambda} \bar{x}_{1}^{2} \\
-\bar{\lambda}
\end{array}\right], \quad \text { with } \bar{\lambda} \bar{x}_{1}>0, \quad(1 / 3) \bar{x}_{1}^{3}=\bar{x}_{2} .
$$

Hence, the domain of definition of $p$ is the set

$$
S=\left\{\left(u_{1}, u_{2}\right) \mid u_{1}>0, u_{2}<0\right\} \cup\left\{\left(u_{1}, u_{2}\right) \mid u_{1}<0, u_{2}>0\right\} .
$$

Notice that this set is nonconvex and disconnected. To each $u \in S$, there corresponds a unique pair $(x, \lambda)$ satisfying (55). Straightforward calculation yields

$$
p(u)=\left\{\begin{array}{lll}
(2 / 3)\left(u_{1}\right)^{3 / 2} /\left(-u_{2}\right)^{1 / 2}, & \text { if } u_{1}>0, & u_{2}<0 \\
(2 / 3)\left(-u_{1}\right)^{3 / 2} /\left(u_{2}\right)^{1 / 2}, & \text { if } u_{1}<0, & u_{2}>0
\end{array}\right.
$$

\footnotetext{
${ }^{3}$ If $M_{\bar{u}}$ is the set of pairs $(x, \lambda)$ corresponding to $\bar{u} \in S$ as in $(57)$, then for any $(\bar{x}, \bar{\lambda}) \in M_{\tilde{u}}$ there exists a sphere $S[(\bar{x}, \bar{\lambda}) ; \bar{\gamma}], \bar{\gamma}>0$, such that $S[(\bar{x}, \bar{\lambda}) ; \tilde{\gamma}] \cap M_{\bar{u}}=\{(\bar{x}, \bar{\lambda})\}$. This follows from the implicit function theorem and implies that $M_{\tilde{u}}$ is a countable set. To see this, pick a vector $\bar{r} \in R^{n+m}$ with rational coordinates in each $S[(\bar{x}, \bar{\lambda}) ; \bar{\gamma}]$ and establish a one-one correspondence of $M_{\bar{u}}$ with a countable subset of $R^{n+m}$.
} 
Example 5.2. For, $n=2$ let

$$
f(x)=f\left(x_{1}, x_{2}\right)=x_{1}+x_{2}, \quad h(x)=\cos x_{1}-x_{2}, \quad \text { for }\left|x_{2}\right| \leq 1 .
$$

Let $\bar{x}=\left(\bar{x}_{1}, \bar{x}_{2}\right)^{\prime}$ be any vector satisfying

Then, (51) is written as

$$
\cos \bar{x}_{1}=\bar{x}_{2} \text {. }
$$

$$
-\bar{\lambda} \cos \bar{x}_{1} z_{1}^{2}>0, \quad \forall z \neq 0, \quad z_{1} \sin \bar{x}_{1}+z_{2}=0 .
$$

The above relation is satisfied for

$$
\bar{\lambda} \cos \bar{x}_{1}<0,
$$

and a conjugate can be defined locally at each point $\bar{u}$ of the form

$$
\bar{u}=\left[\begin{array}{l}
1-\bar{\lambda} \sin \bar{x}_{1} \\
1-\bar{\lambda}
\end{array}\right], \quad \text { with } \bar{\lambda} \cos \bar{x}_{1}<0, \quad \cos \tilde{x}_{1}=\bar{x}_{2} .
$$

Thus, a local conjugate can be defined at any point in the set

$$
S=\left\{\left(u_{1}, u_{2}\right)|| u_{1}-1|<| u_{2}-1 \mid\right\} .
$$

However, to each $u \in S$, there corresponds more than one pair $(\bar{x}, \bar{\lambda})$ satisfying (58). For example, if $\bar{u}=(1 / 2,2)$, then (58) is satisfied for

$$
\bar{\lambda}=-1, \quad \bar{x}_{1}=2 k \pi-\pi / 6, \quad k=\text { integer }, \quad \bar{x}_{2}=\sqrt{3} / 2 .
$$

We have, for the local conjugate at $(2 k \pi-\pi / 6, \sqrt{3} / 2,-1)$, or

$$
p(\bar{u})=\bar{u}^{\prime} \bar{x}-f(\bar{x})=(1 / 2)(2 k \pi-\pi / 6)+\sqrt{3}-(2 k \pi-\pi / 6)-\sqrt{3} / 2,
$$

$$
p(1 / 2,2)=\sqrt{3} / 2-k \pi+\pi / 12,
$$

and thus the value of $p$ depends on the integer $k$, i.e., the point $(\bar{x}, \bar{\lambda})$, which is used in the local definition of $p$.

The following proposition provides essential characterizations of the function $p$. Part (ii) in particular shows that, by conjugation on $\mathrm{p}$, one obtains the original function $\mathrm{f}$ for points near $\overline{\mathrm{x}}$ which lie on the manifold $\{\mathrm{x} \mid \mathrm{h}(\mathrm{x})=0\}$.

Proposition 5.1. Let $f$ be an $h$-locally convex function at $\bar{x}$. Consider a vector $\bar{\lambda}$ satisfying (51), and let $p$ be the $h$-local convex conjugate of $f$ at $(\bar{x}, \bar{\lambda})$ defined by $(56)$. Then,

(i) $\quad p$ is convex and twice continuously differentiable in $S(\bar{u} ; \delta)$ where $\bar{u}$ is given by (52) and, for all $u \in S(\bar{u} ; \delta)$, we have

$$
\begin{gathered}
\nabla p(u)=x(u), \\
\nabla^{2} p(u)=[\tilde{L}(u)]^{-1}-[\tilde{L}(u)]^{-1} \nabla h[x(u)] \\
\times\left\{\nabla h[x(u)]^{\prime}[\tilde{L}(u)]^{-1} \nabla h[x(u)]\right\}^{-1} \nabla h[x(u)]^{\prime}[\tilde{L}(u)]^{-1},
\end{gathered}
$$


where $\tilde{L}(u)$ equals any symmetric invertible matrix such that

$$
\begin{gathered}
\tilde{L}(u) z=L(u) z, \quad \forall z, \quad \nabla h[x(u)]^{\prime} z=0, \\
L(u)=\nabla^{2} f[x(u)]+\sum_{i=1}^{m} \lambda_{i}(u) \nabla^{2} h_{i}[x(u)] .
\end{gathered}
$$

Note that we show that $\nabla^{2} p(u)$ is uniquely defined in this manner. In particular, one may take $\tilde{L}(u)$ equal to $L(u)$, whenever $L(u)$ is invertible.

(ii) Let $\tilde{f}: R^{n} \rightarrow R$ be defined by

$$
\tilde{f}(x)=\sup _{u \in S(\tilde{u} ; \delta)}\left\{x^{\prime} u-p(u)\right\} .
$$

Then, there exists a $\gamma>0$ such that

$$
\tilde{f}(x)=f(x), \quad \forall x \in S(\dot{\bar{x}} ; \gamma), \quad \dot{h}(x)=0 .
$$

Proof. (i) We have, for $u \in S(\tilde{u} ; \delta)$,

$$
\begin{array}{r}
u-\nabla f[x(u)]-\nabla h[x(u)] \lambda(u)=0, \\
h[x(u)]=0, \\
\nabla p(u)=x(u)+\nabla x(u)\{u-\nabla f[x(u)]\},
\end{array}
$$

where the $n \times m$ matrix $\nabla x(u)$ has as columns the gradients of the coordinates of $x$ with respect to $u$. From (65), we obtain

$$
\nabla x(u)\{u-\nabla f[x(u)]\}=\nabla x(u) \nabla h[x(u)] \lambda(u),
$$

while from (66) we have by differentiation

$$
\nabla x(u) \nabla h[x(u)]=0 .
$$

Combining (67)-(69), we obtain

$$
\nabla p(u)=x(y),
$$

and (59) is proved. From the above equality, we also obtain

$$
\nabla^{2} p(u)=\nabla x(u)
$$

Differentiating (65) with respect to $u$, we obtain

$$
I-\nabla \lambda(u) \nabla h[x(u)]^{\prime}-\nabla x(u) L(u)=0,
$$

where $I$ is the $n \times n$ identity matrix, $\nabla \lambda(u)$ is the $n \times m$ matrix having as columns the gradients $\nabla \lambda_{i}(u)$, and $L(u)$ is given by (62). We have

$$
z^{\prime} L(u) z>0, \quad \forall z \neq 0, \quad \nabla h[x(u)]^{\prime} z=0 .
$$


Let $\tilde{L}(u)$ be any symmetric invertible matrix such that

$$
\tilde{L}(u) z=L(u) z, \quad \forall z, \quad \nabla h[x(u)]^{\prime} z=0 .
$$

For example, we can take $\tilde{L}(u)=L(u)$, if $L(u)$ is invertible. Another choice is given by

$$
\tilde{L}(u)=L(u)+r \nabla h[x(u)] \nabla h[x(u)]^{\prime} .
$$

This matrix, in view of (72), is positive definite for $r>0$ sufficiently large by the result of Ref. 6. From (69) and (73), we have

$$
\nabla x(u) L(u)=\nabla x(u) \tilde{L}(u),
$$

and we can write (71) as

$$
I-\nabla \lambda(u) \nabla h[x(u)]^{\prime}-\nabla x(u) \tilde{L}(u)=0 .
$$

Postmultiplying this relation with $[\tilde{L}(u)]^{-1} \nabla h[x(u)]$ and using (69), we obtain

$$
[\tilde{L}(u)]^{-1} \nabla h[x(u)]-\nabla \lambda(u) \nabla h[x(u)]^{[}[\tilde{L}(u)]^{-1} \nabla h[x(u)]=0,
$$

form which

$$
\nabla \lambda(u)=[\tilde{L}(u)]^{-1} \nabla h[x(u)]\left\{\nabla h[x(u)]^{[}[\tilde{L}(u)]^{-1} \nabla h[x(u)]\right\}^{-1} .
$$

Combining (70), (71), (75), (76), we obtain

$$
\begin{aligned}
\nabla^{2} p(u)= & {[\tilde{L}(u)]^{-1}-[\tilde{L}(u)]^{-1} \nabla h[x(u)] } \\
& \times\left\{\nabla h[x(u)]^{\prime}[\tilde{L}(u)]^{-1} \nabla h[x(u)]\right\}^{-1} \nabla h[x(u)][\tilde{L}(u)]^{-1},
\end{aligned}
$$

and the desired relation (60) is proved. In order to show that $p$ is convex in $S(\tilde{u} ; \delta)$, it is sufficient to show that $\nabla^{2} p(u)$ is positive semidefinite. But this follows from (76), since $\tilde{L}(u)$ can be taken to be a positive-definite matrix [for example, of the form (74) for $r$ sufficiently large], and hence $\nabla^{2} p(u)$ is a projection matrix.

(ii) We first observe that we have, for each $u \in S(\bar{u} ; \delta)$,

$$
\nabla\left[x^{\prime} u-p(u)\right]=x-\nabla p(u)=x-x(u) .
$$

Hence, $u$ attains the supremum of $x^{\prime} u-p(u)$ if $x=x(u)$, and it follows that

$$
\tilde{f}[x(u)]=x(u)^{\prime} u-p(u)=x(u)^{\prime} u-u^{\prime} x(u)+f[x(u)],
$$

and finally

$$
\tilde{f}[x(u)]=f[x(u)], \quad \forall u \in S(\bar{u} ; \delta) .
$$

Hence, in order to prove part (ii), it will be sufficient to show that there exists a $\gamma>0$ such that, for each $x \in S(\bar{x} ; \gamma)$ with $h(x)=0$, there is a $u \in S(\bar{u} ; \delta)$ 
such that $x=x(u)$. The set

$$
\{(x, \lambda) \mid[\nabla f(x)+\nabla h(x) \lambda] \in S(\bar{u} ; \delta)\}
$$

is clearly open and contains $(\bar{x}, \bar{\lambda})$. Take $\gamma>0$ such that $(x, \lambda)$ belongs to this set for all $x \in S(\bar{x} ; \gamma), \lambda \in S(\bar{\lambda} ; \gamma)$, and

$$
z^{\prime}\left[\nabla^{2} f(x)+\sum_{i=1}^{m} \lambda_{i} \nabla^{2} h_{i}(x)\right] z>0, \quad \forall z \neq 0, \quad \nabla h(x)^{\prime} z=0,
$$

and furthermore $\gamma \leq \sigma$, where $\sigma$ is the scalar introduced in the application of the implicit function theorem following (54). Let $x$ be such that $x \in S(\bar{x} ; \gamma)$, $h(x)=0$, and let $\lambda$ be any vector in $S(\bar{\lambda} ; \gamma)$. Then, if

$$
u=\nabla f(x)+\nabla h(x) \lambda,
$$

we clearly have $u \in S(\bar{u} ; \delta)$, and $(x, \lambda)$ form a local maximum-Lagrange multiplier pair for problem (53). Since $\gamma \leq \sigma$, by the uniqueness part of the implicit function theorem we obtain

$$
x=x(u), \quad \lambda=\lambda(u) .
$$

We note that it is possible to obtain a local version of Fenchel's duality theorem in terms of local conjugate convex functions. The main idea involved in deriving this theorem can be extracted from the proof of Lemma 3.1. See also Ref. 17.

\section{References}

1. Luenberger, D. G., Introduction to Linear and Nonlinear Programming, Addison-Wesley Publishing Company, Reading, Massachusetts, 1973.

2. FALK, J. E., Lagrange Multipliers and Nonlinear Programming, Journal of Mathematical Analysis and Applications, Vol. 19, pp. 141-159, 1967.

3. LASDON, L., Optimization Theory for Large Systems, The Macmillan Company, New York, New York, 1970.

4. GEOFfrion, A. M., Duality in Nonlinear Programming: A Simplified Applications-Oriented Development, SIAM Review, Vol. 13, pp. 1-37, 1971.

5. EVERETT, H., Generalized Lagrange Multiplier Method for Solving Problems of Optimum Allocation of Resources, Operations Research, Vo1. 11, pp. 399-417, 1963.

6. Debreu, G., Definite and Semidefinite Quadratic Forms, Econometrica, Vol. 20, pp. 295-300, 1952.

7. Bertsekas, D. P., Combined Primal-Dual and Penalty Methods for Constrained Minimization, SIAM Journal on Control, Vol. 13, pp. 521-544, 1975.

8. BertsekAs, D. P., Multiplier Methods: A Survey, Automatica, Vol. 12, pp. 133-145, 1976. 
9. Stephanopoulos, G., and Westerberg, A. W., The Use of Hestenes' Method of Multipliers to Resolve Dual Gaps in Engineering System Optimization, Journal of Optimization Theory and Applications, Vol. 15, pp. 285-309, 1975.

10. RockAfelLAR, R. T., A Dual Approach to Solving Nonlinear Programming Problems by Unconstrained Optimization, Mathematical Programming, Vol. 5, pp. 354-373, 1973.

11. Kort, B. W., and Bertsekas, D. P., Combined Primal-Dual and Penalty Methods for Convex Programming, SIAM Journal on Control and Optimization, Vol. 14, pp. 268-294, 1976.

12. BertseKas, D. P., On the Method of Multipliers for Convex Programming, IEEE Transactions on Automatic Control, Vol. AC-20, pp. 385-388, 1975.

13. RoCkAFEllar, R. T., The Multiplier Method of Hestenes and Powell Applied to Convex Programming, Journal of Optimization Theory and Applications, Vol. 12, pp. 555-562, 1973.

14. RoCKAFELLAR, R. T., Monotone Operators and the Proximal Point Algorithm, SIAM Journal on Control and Optimization, Vol. 14, pp. 877-898, 1976.

15. ROCKAFELLAR, R. T., Augmented Lagrangians and Applications of the Proximal Point Algorithm in Convex Programming, Mathematics of Operations Research, Vol. 1, pp. 97-116, 1976.

16. BERTSEKAS, D. P., On Penalty and Multiplier Methods for Constrained Minimization, SIAM Journal on Control and Optimization, Vol. 14, pp. 216-235, 1976.

17. Bertsekas, D. P., Local Convex Conjugacy and Fenchel Duality, Preprints of 7th Triennial World Congress of IFAC, Helsinki, Finland, 1978; Vol. 2, pp. 1079-1084, 1978. 